\title{
Philosophiques
}

\section{Miguelez, Roberto, La comparaison interculturelle, Montréal, Presses de l’Université de Montréal, 1977.}

\section{Maurice Gagnon}

Volume 6, numéro 2, octobre 1979

URI : https://id.erudit.org/iderudit/203121ar

DOI : https://doi.org/10.7202/203121ar

Aller au sommaire du numéro

Éditeur(s)

Société de philosophie du Québec

ISSN

0316-2923 (imprimé)

1492-1391 (numérique)

Découvrir la revue

Citer cet article

Gagnon, M. (1979). Miguelez, Roberto, La comparaison interculturelle, Montréal, Presses de l'Université de Montréal, 1977. Philosophiques, 6(2),

297-307. https://doi.org/10.7202/203121ar d'utilisation que vous pouvez consulter en ligne.

https://apropos.erudit.org/fr/usagers/politique-dutilisation/ 


\section{ÉTUDES CRITIQUES}

MIGUELEZ, Roberto, La comparaison interculturelle, Montréal, Presses de l'Université de Montréal, 1977.

par Maurice Gagnon

Dense et truffé d'argumentations parfois serrées qu'il est impossible d'analyser ici en détail, ce volume n'est pas facile à résumer. L'auteur consacre son premier chapitre à situer la comparaison interculturelle parmi les différentes méthodes couramment utilisées par la pensée scientifique. Le second chapitre expose les différents problèmes épistémologiques soulevés par l'usage de cette méthode, de même que le postulat empiriste qui la sous-tend. Les trois autres chapitres répondent respectivement aux trois questions suivantes. Comment peut-on délimiter, pour fins de comparaison interculturelle, des unités culturelles clairement et objectivement distinctes? Comment peut-on distinguer, classifier et définir objectivement, et en un système unique, les caractères ou prédicats culturels indispensables à la comparaison? Peut-on vraiment établir, sur une base inductive, des lois réellement universelles dont les comportements individuels et collectifs seront rigoureusement déduits?

La comparaison interculturelle a pour but, nous dit l'auteur, "l'établissement d'hypothèses interculturelles de forme universelle portant sur les rapports existant entre deux ou plusieurs variables, bref l'établissement des lois universelles de la culture ou de la société » (p. 216). Elle est considérée comme possible à la seule condition qu'il existe au moins une variable culturelle commune à plusieurs groupes culturels distincts. D'une façon générale, la comparaison a été dévelop- 
pée en sciences humaines et sociales, surtout en anthropologie, en ethnologie et en sociologie, comme méthode "d'investigation contrôlée " (p. 43) servant de substitut à "l'expérimentation contrôlée " (p. 43), qui est impraticable dans les disciplines précitées en raison de l'impossibilité où se trouve le chercheur d'isoler certaines variables, ou d'en contrôler la variation. Il s'agit en fait de délaisser l'attitude purement descriptive pour faire accéder ces disciplines au statut de sciences authentiques par la formulation de lois générales qui rendent compte du comportement des individus ou des groupes.

Plus précisément, la comparaison interculturelle veut établir qu'une variable commune à plusieurs groupes culturels distincts reste stable ou varie d'un groupe à l'autre en fonction du contexte culturel spécifique à chacun, ou génère d'autres variables dans certains cas. D'après ses premiers utilisateurs empiristes, cette méthode consiste à comparer ces instances «données», « naturelles», que sont les sociétés et les groupes culturels en vue de «l'établissement, par des méthodes surtout quantitatives, d'hypothèses de forme générale portant sur les rapports existant entre deux ou plusieurs variables $\gg(p .45)$. D'un point de vue heuristique, nous dit l'auteur, la comparaison interculturelle sert à découvrir et à formuler le contenu de lois générales; d'un point de vue méthodologique, elle sert plutôt à valider des hypothèses déjà formulées. Le premier point de vue la rend conforme au modèle inductif d'inférence, et on peut alors l'interpréter dans les termes d'une «logique de la découverte». Elle est "nomothétique» (p. 67), et constitue donc une étape immédiatement préparatoire au raisonnement nomologico-déductif. Le second point de vue intègre la comparaison interculturelle au raisonnement hypothético-déductif : elle sert à établir des situations de fait qui confirment ou infirment l'hypothèse-loi, elle fournit la «mineure » de ce raisonnement, selon la forme logique que lui prête Hempel.

Un premier problème que pose la comparaison interculturelle est celui de l'identification des unités à comparer, unités supposément " naturelles », « données » telles quelles à l’observateur intéressé. Pour l'empirisme, ces unités ne doivent 
pas résulter d'un découpage arbitraire et fondé sur des raisons subjectives, mais être objectives. Cela soulève de nombreuses questions sur la signification des termes «naturel » et " donné », et sur les moyens que nous avons pour identifier les unités culturelles « naturelles» dans leur invididualité propre, de même que pour les décrire et les classifier adéquatement.

L'auteur montre qu'il n'existe pas, en sociologie, en anthropologie et en ethnologie pas plus qu'en biolngie, de classification absolument objective d'espèces «naturelles", c'est-à-dire de classification basée sur les caractères à la fois essentiels et distinctifs de chacun des éléments à classifier. Le fait est que nous ne pouvons nous baser que sur les caractères que nous connaissons présentement, d'une connaissance toujours susceptible d'être remise en question, d'être révisée et corrigée. La découverte de nouveaux traits auparavant inconnus peut nous obliger à rejeter complètement la classification déjà établie. Comme cela a été le cas en biologie, le passage de caractères externes et directement observables à des caractères plus «profonds », caractères dont l'existence est postulée dans une théorie, ou dont l'observation n'est possible que grâce à un équipement de laboratoire très spécifique, constitue le passage d'une classification "empirique " à une classification théorique (p. 116-117). Nous quittons alors le réalisme conceptuel naif de la classification des espèces " naturelles" (p. 118-119). Il est clair que la classification présuppose une théorie, et non l'inverse comme le voudrait l'empirisme.

On peut, pour définir et identifier une culture particulière, utiliser divers critères: géographique, politique, linguistique, économique. Aucun de ces critères n'est à lui seul suffisant pour identifier une culture particulière. On peut obtenir des résultats convenables par une combinaison de ces critères, mais il faut bien souligner qu'ils sont tous conventionnels et a priori, et ne découlent pas d'une analyse interne des caractères distinctifs des groupes culturels et de leurs divisions en sous-groupes. Une telle analyse supposerait une étude intensive de chacun des groupes comparés. Mais pour faire cette étude, il faudra d'abord délimiter les groupes, et il n'existe pas de critères sûrs et objectifs pour le faire, comme cela a été déjà montré. Cette étude devrait être intensive, mais 
jusqu’à quel point, jusqu’à quel niveau de particularité ? Devra-t-on examiner un par un tous les individus des groupes concernés ? Où trouver un moyen de choisir les individus les plus représentatifs de leur groupe ? Et si l'étude doit s'arrêter à un certain niveau de généralité, comment choisir ce niveau, c'est-à-dire comment choisir les sous-ensembles auxquels l'étude doit s'arrêter? Les notions implicitement utilisées ici d'unité de comparaison et d'unité d'étude ne sont pas claires, pas facilement applicables, et pas forcément synonymes.

Une autre difficulté vient compliquer encore l'identification des unités culturelles. C'est celle de la continuité structurelle entre les unités culturelles. Deux groupes ont un caractère culturel en commun : est-ce dû à des raisons fonctionnelles, ou à un emprunt d'un groupe à un autre, à l'occasion d'un contact passé ? Si la seconde hypothèse est la bonne, nous n'avons pas deux unités culturelles différentes, mais une seule, tout au moins en autant que ce trait commun, qui sert de point de comparaison, est concerné. Il n'est pas toujours facile de déterminer si deux groupes ont été oui ou non en contact dans le passé : les critères de la proximité et de l'éloignement géographiques ne sont pas décisifs en cette matière. De plus, les deux membres de l'alternative mentionnée plus haut ne sont pas mutuellement exclusifs : un emprunt fait à une autre société peut aussi répondre à un besoin fonctionnel, remplir dans le groupe emprunteur une fonction auparavant inexistante, mais dont le besoin se faisait sentir, ou une fonction auparavant remplie avec moins d'efficacité ou de degré de satisfaction. La question est de savoir quand l'emprunt a été fait et pourquoi, et plus précisément pourquoi ce trait a été emprunté parmi d'autres possibles. La complexité des rapports sociaux et des faits historiques ne nous permet jamais de trouver une réponse claire et indubitable à ces questions. Il faut ajouter à ceci que les éléments empruntés sont intégrés, et, par conséquent, la plupart du temps modifiés par la culture emprunteuse. Cela veut dire en fait que les relations purement diffusionnelles ou purement fonctionnelles sont sans doute rares, les secondes étant de toute façon techniquement invérifiables. 
Ces considérations étant faites, Miguelez montre que le problème de la continuité structurelle change de visage. Il ne s'agit plus d'établir l'identité, le caractère distinctif et l'indépendance absolue de divers groupes culturels, mais plutôt de trouver l'interprétation des corrélations observées. Dans plusieurs groupes culturels, deux traits culturels peuvent être fortement ou faiblement, positivement ou négativement, associés. Mais il n'existe pas de méthode pour établir avec certitude si l'association ou l'absence d'association est réelle ou fortuite; si elle est due à des emprunts historiques ou à des facteurs de fonctionnalité interne qui font que l'un des deux traits découle nécessairement de la présence de l'autre ; et dans le cas d'une relation causale, si elle est indirecte ou directe, c'est-à-dire admet ou non des intermédiaires entre la cause et l'effet.

Dans un monde où le prosélytisme religieux et idéologique est très actif, où des communications de plus en plus rapides favorisent les échanges culturels à l'échelle planétaire, il est illusoire de prétendre dresser une classification des cultures en unités naturelles et objectives, sur la base de données «brutes". Le découpage est relatif à un ensemble plus ou moins conscient, plus ou moins articulé de concepts et d'hypothèses a priori. On peut bien sûr, par des méthodes statistiques, établir divers types de corrélations entre des variables culturelles et montrer comment ces corrélations évoluent dans le temps. Mais il est impossible de décider, à la limite, si une société soumise à l'influence d'une autre a évolué tout en conservant son identité profonde, ou si elle a été assimilée, absorbée par l'autre. Il reste simplement possible de repérer des groupes géographiquement localisés où certains traits culturels déterminés sont plus fortement marqués que dans tous ou la plupart des autres.

Une autre série de problèmes concerne le découpage des attributs, prédicats, variables ou propriétés culturelles appartenant à des groupes culturels et servant de point de comparaison interculturelle. Les difficultés sont ici d'ordre sémantique. Le Outline of Cultural Materials est le résultat d'une tentative pour dresser une classification exhaustive de toutes les unités conceptuelles qui s'appliquent effectivement à au moins 
un groupe culturel. Il a été dressé pour des raisons pragmatiques, c'est-à-dire pour épargner du temps, des efforts et des fonds aux chercheurs. Sa fabrication suppose un choix dans l'inventaire des unités conceptuelles, choix qui nous renvoie à des critères a priori. L'inventaire descriptif originel est fait le plus souvent par des non-spécialistes venant d'horizons culturels très différents, et il n'y a pas de description purement objective sans interprétation. Toutes les précautions ultérieures (codage, par un spécialiste, du matériel rassemblé, puis vérification par un second spécialiste) n'effacent pas l'hétérogénéité des multiples codages initiaux. En supposant qu'on peut mettre sur pied un système unique, universel et complet de catégories culturelles fondées sur le sens commun, les promoteurs du Outline of Cultural Materials font un postulat énorme, qui exprime et contredit à la fois leur empirisme. Le danger ici est que les catégories se substituent au réel. En fait, on a fondé sur des raisons pratiques un système unique de classification, lequel fonde à son tour un ensemble de catégories universelles de la culture. Pourtant, cette dernière relation est l'inverse de ce qu'elle devrait être en bonne logique. L'explication de cette inversion, estime avec raison Miguelez, c'est qu'il n'existe pas de théorie des catégories universelles de la culture qui puisse rallier l'assentiment de la majorité des chercheurs et fonder scientifiquement un système unique de classification. Le fondement pragmatique mentionné plus haut ne crée pas la neutralité des observateurs et des chercheurs.

Une autre difficulté est celle de l'univocité des significations. Une propriété $S$, commune aux unités culturelles $A$ et $B$, est-elle bien la même dans les deux cas, en dépit des contextes différents, des séries différentes d'autres propriétés auxquelles elle est reliée dans chaque cas? Dire que chaque unité culturelle est un tout unique et incomparable implique le renoncement à la découverte et à l'usage de lois en sciences humaines et sociales. On peut dire aussi que la comparaison interculturelle exige une équivalence des signifiés (i.e. les contenus sémantiques des signes), non une équivalence des signifiants (i.e. la manière matérielle dont le signifiant se manifeste). On peut s'assurer, par une analyse interne des cultures concernées, de l'équivalence des signifiés, mais alors il 
faut renoncer à la comparaison interculturelle universelle et aux catégories universelles de la culture pour se contenter de la comparaison restreinte, à moins de postuler tout simplement la validité du codage utilisé pour identifier et classer les unités culturelles et conceptuelles.

L'équivalence des significations ne peut être ramenée à l'équivalence des stimuli, car les mêmes stimuli, les mêmes méthodes, voire les mêmes instruments, ne provoquent pas toujours les mêmes résultats ou les mêmes interprétations, surtout en sciences sociales, en raison des différences de contexte et d'observateur, et en raison du caractère plus difficilement mesurable des phénomènes. Il faut bien connaître les cultures comparées pour garantir l'équivalence des significations utilisées en vue de la comparaison interculturelle, et cette dernière a justement pour but de mieux nous faire connaître ces cultures. Il n'est guère plus efficace de ramener l'équivalence des significations à celle des inférences réalisées à partir des observations. Cette hypothèse nous entraîne dans des discussions sans issue claire sur les rapports entre les indicateurs observables et les propriétés cachées qu'on peut en inférer. On sait qu'en cette matière, il n'y a pas de système de relations biunivoques. Des indicateurs différents peuvent nous renvoyer à une même propriété cachée, ou l'inverse. Les ensembles d'indicateurs appartenant à des groupes ou sans groupes culturels différents ne sont pas forcément disjoints, et il faut, sur la base de critères a priori et variables, faire un choix d'indicateurs parmi les propriétés observables. Il n'y a pas d'équivalence absolument sûre et claire des significations. Ici encore, le chercheur doit se contenter d'approximations.

Une troisième série de questions tourne autour des résultats effectifs de la comparaison interculturelle: a-t-elle produit des lois universelles de la culture et de la société ? Un examen attentif nous révèle que ces lois sont ou bien vraies et non universelles, ou bien fausses et universelles, ou bien universelles et probablement vraies, mais triviales. Les limites du découpage des unités culturelles et des unités conceptuelles ne sont pas étrangères à cette situation. L'indépendance des unités culturelles garantit la validité des cas qui confirment l'hypothèse interculturelle, mais leur dépendance n'est pas 
suffisante pour expliquer les cas négatifs, ceux qui infirment l'hypothèse. Les questions sur le pourquoi de l'emprunt, sur les raisons qui ont déterminé tel emprunt plutôt que tel autre, et sur les facteurs qui ont maintenu intact un trait emprunté nous renvoient à une analyse contextuelle de la culture concernée. À l'inverse, le découpage des unités conceptuelles permet d'expliquer les cas négatifs, mais n'a aucun impact sur les cas positifs. Un plus grand nombre de catégories (donc un découpage plus raffiné) a tendance à faire augmenter les exceptions, mais aussi les exceptions expliquées : la multiplicité des codes utilisés lors de la classification fait augmenter les exceptions, mais le repérage de ces divers codes permet de les expliquer. Les facteurs de multicausalité (plusieurs causes doivent nécessairement concourir à la production d'un effet) et de pluricausalité (le même effet peut être produit par plusieurs causes différentes et agissant chacune de façon indépendante des autres) sont aussi sources d'exceptions explicables quand on a compris les mécanismes en question.

S'interrogeant ensuite sur l'interprétation des corrélations, l'auteur montre qu'il est impossible, sans recourir à des hypothèses, soit du sens commun, soit de nature scientifique, de décider si une association régulière entre variables est réelle ou fortuite, causale (asymétrique) directe ou indirecte. Il s'ensuit que la comparaison interculturelle ne fonde pas un modèle, mais bien l'inverse (p. 249). Si le modèle est une supposition empirique du sens commun, l'interprétation de la corrélation est subordonnée à une hypothèse imprécise et d'un bas niveau théorique. Et comme ces suppositions empiriques ne sont pas toujours explicites, le danger est grand que l'interprétation des corrélations soit biaisée par des préjugés issus de la culture du chercheur.

Comme il semble que la comparaison interculturelle restreinte est la seule qui soit méthodologiquement acceptable, la seule qui fournisse des garanties acceptables d'équivalence des significations, Miguelez se demande s'il est possible de passer de cette comparaison à la comparaison universelle. Il montre que ce passage augmente le nombre de variables libres dans les énoncés, et qu'il implique un processus où des noms propres de systèmes individuels sont remplacés graduellement 
par des noms de variables (concepts de classes). C'est en fait un "processus de généralisation conceptuelle correspondant à, et dépendant d'un processus de généralisation empirique » (p. 264). L'hypothèse devient ainsi de plus en plus générale, "superficielle » et vague, car ainsi on passera d'une corrélation entre, par exemple, «mobilisation politique » et « exposition à la radio " à une corrélation entre «mobilisation politique» et « exposition aux mass media », et ainsi de suite. L'hypothèse perd graduellement en profondeur et à la limite elle devient triviale.

Les conclusions que pose Miguelez sont prévisibles, à la fin de l'exposé. La comparaison interculturelle a donné des résultats très décevants en regard des espoirs qu'on avait placés en elle. Ceci n'est pas dû à la complexité des faits humains et sociaux, ni à des déficiences méthodologiques ou techniques, mais plutôt au fait que la comparaison interculturelle est fondée sur un modèle de science - le modèle empiriste trop pauvre et trop simpliste. Miguelez suggère que les sciences humaines et sociales doivent plutôt s'aligner sur un modèle de science où l'objet est conçu comme construit et non comme naturel ou donné, un objet qui est en état de rupture avec l'objet donné, tout en ayant un référent réel dont il révèle les conditions d'existence. La comparaison interculturelle restera sans doute possible dans ce modèle, mais avec un rôle et des objets différents. Une telle science sociale ne sera pas, comme la science empiriste, le simple redoublement sophistiqué et idéologique des sociétés ou des cultures, et elle exercera une réelle fonction vraiment cognitive.

En plus de constituer une analyse extrêmement fouillée et perspicace de la comparaison interculturelle, ce volume est aussi une critique très sévère, mais aussi très juste, de la conception empiriste de la connaissance. Les seuls reproches que nous adresserions à l'auteur sont de l'ordre de la présentation. On aimerait de la part de l'auteur un peu plus d'empressement à définir clairement les termes qu'il utilise. En particulier, il emploie les expressions «loi », "théorie » et « modèle " dans des sens qui semblent parfois - mais pas toujours - synonymes. On est surpris de lire à la p. 172 que les éléments d'une théorie sont des propositions empiriques. 
Mais une théorie, qui parle ordinairement d'entités théoriques, ne doit-elle pas comporter aussi d'autres types de propositions ? Et à la p. 173 , on lit : «... dans une systématique scientifique le cadre des concepts n'est pas indépendant d'une théorie, c'est-à-dire d'une construction empirique ». Nous sommes portés à croire qu'une construction d'entités théoriques n'a rien d'empirique, et qu'un énoncé empirique est un énoncé descriptif d'une expérience réelle. Nous aurions donc apprécié que l'auteur explique ce qu'il entend par « construction empirique». L'auteur parle souvent aussi de modèle : modèle inductif de la science, modèle empirique. À la p. 248 , il nous parle des suppositions empiriques de la science ou du sens commun, qui servent à fonder la signification de relations statistiques (en l'occurence une corrélation indirecte) tout en étant logiquement indépendantes de la relation concernée. À la page suivante, il est dit: "l'interprétation d'une relation empiriquement constatée est subordonnée logiquement aussi bien que dans la pratique à une conjecture dont la formulation ne peut pas . . . être l'aboutissement d'un processus seulement inductif. En d'autre termes,... ce n'est pas encore la comparaison qui fonde le "modèle ", mais bien le « modèle » qui fonde la comparaison. " Ainsi donc, la supposition ou conjecture est modèle. Doit-on croire que les termes hypothèse, théorie, supposition, modèle sont tous synonymes ? Pourtant, il existe déjà un certain usage que l'auteur aurait pu respecter. Quand on parle du modèle d'atome de Bohr, par exemple, on se réfere à une représentation concrète, bien qu'hypothétique, de la structure matérielle de l'atome; alors que la théorie atomique qui accompagnait ce modèle était tout à la fois un ensemble d'énoncés théoriques et un système d'équations. Et le mot théorie désigne plutôt une supposition théorique largement acceptée dans la communauté scientifique parce qu'elle jouit d'un degré élevé de confirmation, par opposition à une hypothèse.

Quand l'auteur se demande (p. 278) si des comparaisons inter-culturelles auront droit de cité dans une science sociale travaillant sur des objets construits, nous croyons que sa réponse aurait dû être plus fermement affirmative, car l'impossibilité de l'expérimentation contrôlée existera encore dans 
cette nouvelle science. Il a raison de dire que la comparaison interculturelle y jouera un autre rôle, mais nous aurions aimé qu'il esquisse la nature de ce rôle. Pour notre part, nous croyons que la comparaison servira à confirmer ou infirmer des hypothèses explicatives des corrélations ou absences de corrélations entre variables, toujours dans les limites d'un contexte déterminé.

L'auteur parle de « logique de la découverte » quand il interprète la comparaison interculturelle comme un type d'induction. Sans insister ici sur les questions et les difficultés que soulève cette expression de «logique de la découverte", nous signalons que l'auteur aurait pu les éviter en formulant ses propos en termes de «contexte de découverte» et " contexte de justification ", qu'on retrouve dans des études récentes.

Ce livre, pour la compréhension qu'il nous apporte de la comparaison en sciences sociales, et pour sa critique pénétrante de l'empirisme, mérite grandement d'être lu. Sous la sécheresse technique et logique de l'exposé, on trouve une analyse intelligente et perspicace.

Université de Sherbrooke 